\title{
The Hopkins Verbal Learning Test had high sensitivity and good specificity for detecting mild dementia in older adults
}

Frank RM, Byrne GJ. The clinical utility of the Hopkins Verbal Learning Test as a screening test for mild dementia. Int J Geriatr Psychiatry 2000 Apr;15:317-24.

QUESTIONS: Is the Hopkins Verbal Learning Test (HVLT) a reliable and valid screening test for mild dementia in older adults? How does it compare with the Mini-Mental State Examination (MMSE)?

\section{Design}

Blinded comparison of HVLT and MMSE results with Diagnostic and Statistical Manual of Mental Disorder, Fourth Edition (DSM-IV) diagnoses of dementia (the diagnostic standard).

\section{Setting}

The Geriatric Psychiatry Service of the Royal Brisbane Hospital and District Health Service, Australia.

\section{Patients}

56 patients (mean age 75 y, 63\% women, mean education 8.5 y) participated. Exclusion criteria were age $<65$ years, hearing impairment, aphasia, MMSE score $<18$, insufficient ability to speak English, or inability to consent.

\section{Description of tests and diagnostic standard}

3 experienced registered nurses blinded to patients' diagnostic status administered the HVLT and the MMSE. The maximum HVLT total score was 36 and for the HVLT recognition score, 12. The maximum MMSE total score was 30 . Each patient was assessed by an independent psychiatrist (blinded to HVLT and MMSE test results) for the presence of dementia and other psychiatric diagnoses using $D S M-I V$ criteria.

\section{Main outcome measures}

Area under the receiver operating characteristic (ROC) curve, sensitivity, specificity, positive and negative likelihood ratios, and inter-rater reliability.

\section{Main results}

26 patients had DSM-IV dementia, 15 had psychiatric diagnoses other than dementia, and 15 were normal control patients. The area under the ROC curve for both the HVLT and the MMSE was 0.93. Using ROC analysis, the optimal cut point for detecting dementia with the HVLT was $18 / 19$; for the MMSE it was $25 / 26$. The table shows the sensitivity, specificity, and positive and negative likelihood ratios for each. The HVLT had better sensitivity than did the MMSE, but the MMSE had higher specificity. Interrater reliability was high $(>0.99)$ and comparable for the 2 tests. Both HVLT and MMSE scores were positively correlated with education level.

\section{Conclusion}

The Hopkins Verbal Learning Test had high sensitivity and good specificity for detecting mild dementia in older people.
Test characteristics for detecting mild dementia* glossary; Cls and LRs calculated from data in article.

\section{COMMENTARY} collateral history or informant questionnaire. tice: toward a consensus. Alzheimer Dis Assoc Disord 1998;12:1-13. J Neurol Neurosurg Psychiatry 1999;66:177-83.
Source of funding: not stated.

For correspondence: Dr G Byrne, Department of Psychiatry, University of Queensland, K Floor, Mental Health Centre, Royal Brisbane Hospital, Queensland 4029, Australia. Fax +61733655488 .

A modified version of this abstract also

appears in

Evidence-Based

Nursing.

\begin{tabular}{lllll} 
Scale, cut point & Sensitivity $(95 \%$ Cl) & Specificity (CI) & +LR & -LR \\
\hline HVLT, $18 / 19$ & $96 \%(80$ to 100$)$ & $80 \%(61$ to 92$)$ & 4.80 & 0.05 \\
\hline MMSE, $25 / 26$ & $88 \%(67$ to 98$)$ & $93 \%(78$ to 99$)$ & 12.57 & 0.13 \\
\hline
\end{tabular}

${ }^{*}$ HVLT $=$ Hopkins Verbal Learning Test; MMSE $=$ Mini-Mental State Examination. Diagnostic terms defined in

The prevalence of dementia ranges from $6 \%$ to $10 \%$ in people $\geqslant 65$ years of age, an expanding percentage of the population. Recent pharmacotherapeutic and genetic advances have highlighted the importance of early diagnosis. Because the diagnosis is often missed, valid and reliable diagnostic screening instruments are needed. Targeted screening of at risk groups allows secondary prevention measures to be initiated. ${ }^{1}$

In a relatively small sample that was purposely selected to include patients with mild dementia, Frank and Byrne compared the HVLT with the MMSE. Their study suggests that with the HVLT limitations imposed by such confounding variables as age do not occur, and in the case of educational level, they are less problematic. These findings warrant further evaluation in a larger sample size. Low educational level is a likely risk factor for dementia, ${ }^{2}$ which potentially complicates the picture. The comparatively greater sensitivity and lesser specificity of the HVLT suggest that more false-positive results occur with that test, and the positive likelihood ratio is less than that of the MMSE. Using the suggested cut point scores in this study by Frank and Byrne, the MMSE is better at ruling out a diagnosis of dementia, whereas the HVLT is better at detecting it. The availability of 6 equivalent forms of the HVLT offers the potential to reduce learning bias during repeat assessments. The HVLT is therefore a potentially useful adjunct in assessing dementia, but the test requires further evaluation in larger, longitudinal studies and in those of different cultures. Results from cognitive screening must be interpreted in context; for example, cognitive deficit can also occur in delirium. The diagnosis of dementia therefore requires evaluation of the composite clinical picture, including a

Peter G Lawlor, MB, MMedSc University of Alberta Edmonton, Alberta, Canada

1 Brodaty H, Clarke J, Ganguli M, et al. Screening for cognitive impairment in general prac-

2 Letenneur L, Gilleron V, Commenges D, et al. Are sex and educational level independent predictors of dementia and Alzheimer's disease? Incidence data from the PAQUID project. 